\title{
Realtime System Control by means of Path Expressions
}

\author{
Albert L. Schoute
}

Jaap Jan Luursema

University of Twente, Department of Informatics, P.O. Box 217, 7500 AE Enschede, Netherlands

\begin{abstract}
This paper treats a high-level, algebraic programming method for the on-line control of actions in a realtime, parallel processing environment. The method is based on interaction of path expressions. On the basis of a set of path expressions evocation of actions can be controlled in realtime in a fully automated way.

It is shown how intelligent system behaviour can be obtained by a combination of rules given as path expressions. Each of
these rules specifies some partial behaviour to which the system must comply. The control system operates as a rule-based action planning system that works on-line in an asynchronous environment.
\end{abstract}

\section{INTRODUCTION}

In this paper we describe an algebraic programming method for the high-level control of actions in a realtime, parallel processing environment. The aim of the paper is to present this method by means of examples.

The programming method is based on path expressions [1]. Path expressions can be considered as a generalisation of regular expressions. They define a set of possible evolutions over an alphabet of operations or actions, just like regular expressions define a set of possible strings over some alphabet of symbols.

On the basis of a set of path expressions the evocation of actions can be controlled in realtime in a fully automated way. A prototype of such a controlling system has been developed and is described in this paper.

The selection of actions is dependent on the way path expressions are combined. Path expressions can be considered as rules that specify some partial behaviour to which the system must comply. The total system behaviour is obtained as a result of the inference of these behavioural rules. In some sense the control method may be viewed as a rule-based expert system, which operates as an on-line action planning system. The set of path expressions constitutes the knowledge base. The inference mechanism is however unusual. It is based on the notion of intersection of path expressions. The intersection defines a combined evolution that satisfies each of the individual path expression components.

After a short overview of the path expression syntax in section II, the method of programming with path expressions is explained in section III by a simple example. We show how system behaviour in a asynchronous processing environment can be specified this way. In section IV main features of the prototype control system that has been built, are exposed. The modelling aspect of high-level, realtime control is treated more extensively in section $V$. Experiences with the control method and the prototype system are summarized in the concluding section VI.

\section{Path ExPression CONSTRUCTS}

The basic path expressions constructs that we will consider are: sequential composition (using the sequence operator ';') parallel composition (using the parallel operator ' $\&$ ') alternative composition (using the choice operator ','). Extended path expression constructs are the combinatory composition or intersection (using the operator ' $*$ ') and repetitive forms of compositions as shown in table 1.

\begin{tabular}{|ll|}
\hline Empty path & nil \\
Single action & $\mathrm{a}$ \\
Sequence & $\mathrm{P} 1 ; \mathrm{P} 2$ \\
Alternative & $\mathrm{P} 1, \mathrm{P} 2$ \\
Parallel & $\mathrm{P} 1 \& \mathrm{P} 2$ \\
Intersection & $\mathrm{P} 1 * \mathrm{P} 2$ \\
Sequential repetition & $\{\mathrm{P}\}$ \\
Parallel repetition & $\{\mathrm{i}=1 \ldots \mathrm{n}: \mathrm{Pi}\}$ \\
Repetitive alternative & $[\mathrm{P}]=1 . . \mathrm{n}: \mathrm{Pi}]$ \\
Repetitive intersection & $* \mathrm{i}=1 . \mathrm{n}: \mathrm{Pi}>$ \\
Conditional path & IF $=1 \ldots \mathrm{n}: \mathrm{Pi}$ \\
& IF pred THEN P \\
Activator path & $\mathrm{P} !$ \\
Acceptor path & $\mathrm{P} ?$ \\
\hline
\end{tabular}

Table 1. Summary of path expression constructs

The repetitive constructs are either bounded by an index range $\mathrm{i}=1 . . \mathrm{n}$, or - in case of sequential and parallel repetition unbounded, which means zero or more repetitions.

The meaning of the different path expressions constructs will become clear in the course of this paper. We start with an simple example to help getting a basic understanding.

\section{SYSTEM SPECIFICATION EXAMPLE}

As an illustration of path expression construction consider a system of traffic lights for a two road junction. Its basic, yet unsynchronized, operational behaviour can be formulated as:

\{green(1);yellow(1);red(1)\} \& \{green(2);yellow(2);red(2)\} or more compact by:

$[\mathrm{i}=1 . .2:\{$ green(i);yellow(i);red(i) $\}]$ 
The action names green(1), yellow(1), etc are considered as representing basic actions defined by procedures at an underlying level. In the example they are supposed to make the light green, yellow, etc.

\section{A. Constraining system behaviour}

The traffic lights need to be synchronized by additional restrictions. For example, a safety rule may allow a light to become green (and yellow afterwards) only if the other light is red. This exclusion property can be expressed as a choice within a sequential repetition:

\{(green(1);red(1)), (green(2);red(2))\}

or more compact:

$$
\{<\mathrm{i}=1 . .2: \operatorname{green}(\mathrm{i}) ; \operatorname{red}(\mathrm{i})>\}
$$

At the beginning of each cycle path expression P2 will allow either green(1) or green(2). (It is assumed that initially, and after each cycle, both lights are red.) However, when say traffic light 1 becomes green by action green(1), P2 only allows action red(1), whereas it will inhibit green(2) (and also red(2)) It should be noted that path expressions only restrict actions of which the name appears in the expression. Hence the action yellow(1) or yellow(2) is not constrained by P2.

The synchronized behaviour is obtained by combining both path expressions P1 and P2. (Formally, we write this as $\mathrm{P} 1 * \mathrm{P} 2$, where * denotes the intersection operator. The semantics of the intersection operator is described more precisely in [4].)

Multiple path expressions combined in an intersection have an evolution that obeys all path expressions. An action can only be executed if all path expressions agree. Intersecting path expressions interfere on the basis of common action names. It may be verified that a sequential evolution like

$$
\begin{aligned}
& \text { green }(2) \rightarrow \text { yellow }(2) \rightarrow \operatorname{red}(2) \rightarrow \\
& \text { green }(1) \rightarrow \text { yellow }(1) \rightarrow \operatorname{red}(1) \rightarrow \\
& \text { green }(1) \rightarrow \text { yellow }(1) \rightarrow \operatorname{red}(1)
\end{aligned}
$$

satisfies both path expressions.

Sets of path expressions that interfere as described above, are useful to co-ordinate the occurrences of actions on a high level This co-ordination can be effectuated by a run-time mechanism that keeps track of the evaluation state of the expressions (see section 4.3 on implementation aspects).

Apart from this, the actions itself may of course interact too at a lower level according to the application specific state. The extent to which the operational behaviour of a system is controlled by path expressions may vary. It will depend on the level at which actions are considered as elementary, selfcontained steps in respect to the total system behaviour.

Timing aspects, for example, may remain hidden within the procedures, which implement the actions. The duration of the green, yellow and red phases could be determined at a low level, possibly influenced by traffic detectors.

However, in many cases timing decisions are part of globa strategies and can not be separated from the high level control.

\section{B. Realtime strategies}

The path expression specification language has been extended with several features to facilitate more complex realtime control at the path expression level.

Actions may be parametrized - not only by index-variables but also by other parameters. For example, the length of the yellow-phase could be specified in time units by, say, yellow $(1,20)$ and yellow $(2,15)$.

An important extension concerns the use of conditional path expressions by which the execution becomes dependent upon predicates [2]. Predicates, which are boolean expressions, have the effect of a guard: the evaluation of a conditional path is postponed as long as the predicate is false. This may of course lead to a selection of an alternative subexpression. Such a choice is given implicitly in case of the IF-THEN-ELSE construct. The expression

\section{IF pred THEN P1 ELSE P2}

must be read as

\section{(IF pred THEN P1, IF NOT pred THEN P2)}

Predicate expressions may contain relations between variables. Two kinds of special variables: event counters and time stamps are system defined and associated with each action name:

1. Two standard event counter variables denoted by ACT_name and TERM_name give the number of activations, respectively terminations, of the specified action that have taken place during system history.

2. Similarly, two standard time stamp variables denoted by TACT name and TTERM name give the time instant of last activation, respectively termination, of the specified action (measured in system ticks).

Predicates with time stamps may be constructed using the following relational operators:

\section{time stamp var LAT / SAT time constant}

\section{time stamp var BEFORE / AFTER time stamp var}

The first type of comparison is true if the time stamp variable reflects an instant which happened longer ago than (LAT) or shorter ago than (SAT) the given amount of time.

In case of our traffic lights, using conditional path expressions one may state additional timing rules. The following rule specifies the duration of the green-phase:

$\{\mathrm{i}=1 . .2$ : IF TACT_green(i) LAT 30 THEN yellow(i) $\}$ (P3)

The yellow-action is only allowed when the activation of the green-action has occurred longer ago than 30 time units.

For safety reasons one may also want to state a minimal period during which lights must have been red before any of the lights may jump to green:

$\{$ IF TACT_red(1) LAT 5 THEN $<\mathrm{i}=1 . .2: \operatorname{green}(\mathrm{i})>$ \} (P4)

$\{$ IF TACT_red(2) LAT 8 THEN $<\mathrm{i}=1 . .2: \operatorname{green}(\mathrm{i})>$ \} (P5)

Apart from relations also predicate functions may be part of predicate expressions. Predicate functions are boolean function procedures which test application dependent conditions. 
Predicate function values may change as a result of asynchronously occurring events or interrupts.

The time during which a traffic light stays green may be prolonged, if traffic still passes the junction, up to some maximum:

$$
* \mathrm{i}=1 . .2 \text { : }
$$

( IF no_traffic(i) THEN yellow(i)

$$
\text { IF TACT_green(i) LAT } 90 \text { THEN yellow(i) ] }
$$

\section{Matching of activators and acceptors}

A new aspect with respect to path expression control concerns the qualification of action names as being either an activator or an acceptor. (The actual qualification depends on the fact whether the action itself or an enclosing (sub)expression is defined as activator or as acceptor, see table 1.) By introducing this difference better control can be achieved over the allowed or desired evolutions. Also in real life the difference between being prepared to perform an action or to tolerate an action has a profound influence on the world's evolution. The precondition for the activation of an action is an enabled activator of the action in some path and no disabled acceptors of the action in other paths. The absence of an acceptor in a path for some action must be interpreted as a "don't care".

Occurrences could be considered as the outcome of a multiway synchronization between actions with the same name in different paths. We require that exactly one of the participants of the multi-way synchronization is an activator.

It is natural to take path $\mathrm{Pl}$ of the example as activator because it reflects the actual mechanism. Path expressions P2 up to P6 specify restrictions and should be taken as acceptors. However, within a path expression mixtures of activators and acceptors may appear. Error situations could for instance generate actions:

$$
<\mathrm{i}=1 . .2 \text { : }
$$

$$
\text { IF TTERM_green(i) LAT } 2000 \text { THEN alarm! > }
$$

\section{A PROTOTYPE SYSTEM}

In order to experiment with the method of path expression based on-line control we developed a prototype system named PBOS (Path Based Operating System). It has been built on top of a multitasking system on the IBM/PC written in TURBOPascal.

The idea of PBOS is to evoke and control all actions within the system by means of path expressions. Actions mentioned in these path expressions are basic system commands or application dependent tasks that can be specified at the user command level.

Path expressions can be entered interactively, either as commands (treated as activator) or as rules (treated as acceptor). They are compiled into a binary parse tree representation. The collection of path expressions that at any moment controls the system behaviour is called the rulebase. A run-time mechanism determines which actions are enabled or disabled according to the evaluation state of the path expressions in the rulebase. If an activator is enabled in some path and the action is not disabled by any other path, it will be activated.
Some features of PBOS are described in more detail in this section.

\section{A. The PBOS command shells}

The special feature of command shell processes in PBOS is that every command line given as input is treated as a path expression. Hence, the command shell language conforms to the path expression syntax. This offers a powerful way to structure single actions into compound commands. Actions in a command are activators by default. If a command is successfully compiled, it is added to the rulebase. As a result, the actions of the command will be executed (of course only as far as the other rules allow that). After termination of the command, it is removed again from the rulebase. The execution of an action in a command leads to the forking of the appropriate task. Actions may correspond to standard system tasks or to application tefined tasks.

Special system tasks exist to add path expressions as rules to the rulebase, to remove and to modify them. Table 2 gives an overview of all system defined actions in PBOS. Actions that are unknown are simulated by a process that prints a start message, sleeps a (small) random time and then prints a stop

\begin{tabular}{|c|c|}
\hline $\begin{array}{l}\text { pstate } \\
\text { shell } \\
\text { trans } \\
\text { sleep } \\
\text { nrule } \\
\text { arule } \\
\text { drule } \\
\text { erule }\end{array}$ & $\begin{array}{l}\text { show status of processes } \\
\text { start new shell } \\
\text { transmit form source to destination } \\
\text { delay executing process } \\
\text { define new set of rules } \\
\text { add rules to existing set } \\
\text { delete all rules } \\
\text { edit individual rules by subcommands: } \\
\text { add, edit, del, reset, list }\end{array}$ \\
\hline Irule & list all rules \\
\hline $\begin{array}{l}\text { Icom } \\
\text { dcom }\end{array}$ & $\begin{array}{l}\text { list all unfinished commands } \\
\text { delete a unfinished command }\end{array}$ \\
\hline Inames & $\begin{array}{l}\text { list action/macro names and their associated } \\
\text { standard variables }\end{array}$ \\
\hline
\end{tabular}
message.

The control system is very rigourous in the sense that also these system actions have to obey the rules in the rulebase.

Table 2

Standard system actions in PBOS

In the following example we first define a rulebase (with only

\begin{tabular}{|c|c|}
\hline sh) nrules & $\begin{array}{l}\text { Command to define a new rulebase. } \\
\text { 'sh)' is the prompt of the shell. }\end{array}$ \\
\hline nr) $\{$ a ; Irules! ; b \} & The new rule. \\
\hline $\mathrm{nr})^{\wedge} \mathbf{Z}$ & $\begin{array}{l}\text { 'nr)' is the prompt of nrules. } \\
\text { Typing new rules is terminated by } \\
\text { a control-Z }\end{array}$ \\
\hline $\begin{array}{l}\text { sh) Irules } \\
\text { \{ a ; Irules! ; b \} } \\
\text { sh) a \& b }\end{array}$ & $\begin{array}{l}\text { Command to list the rulebase. } \\
\text { Output of lrules. } \\
\text { User command: a rule '(a\&b)!' is } \\
\text { temporarily added to the rulebase. }\end{array}$ \\
\hline Start a & $\begin{array}{l}\text { Action 'a' is executed. Since action 'a' } \\
\text { is unknown, it is simulated. The system }\end{array}$ \\
\hline
\end{tabular}
one rule) and list it. Then we supply a command that interferes with the defined rule.

Input typed by the user is printed bold. 
Stop a

prints start and stop messages. Action ' $b$ ' is not yet executed, according to the rule.

$\{\mathrm{a} ;$ lrules! ; b \}

The action lrules is executed as side effect of the command, according to the rule in the rulebase.

Start b Stop b

Now, action ' $b$ ' is executed.

After termination of ' $b$ ', the rule

'(a\&b)!' is removed from the rulebase.

In PBOS, new shells can be created with the system action 'shell'. The shells are organized in a family tree. Every shell corresponds to a node in the tree and has its own set of rules. A shell has to obey these rules, but also the rules defined by nodes higher in the tree. Alternatively: rules defined by some shell also have to be obeyed by all its subshells, by their subshells etc.

This means that rules defined by the root shell apply to all shells. The root shell is therefore the most powerful shell.

Assume for example that the following rule is defined by the root shell:

\{ nrules, lrules \}

As a result of this rule, no two shells can ever define new rules or list their rules at the same time.

\section{B. Macro extension}

We mention here the possibility to define a path expression as macro by a line:

name $=$ path expression

This means that a path expression can be given a name and that this name may be used as a short-hand instead of supplying the whole expression every time. It even means that recursion is introduced in the language, for instance by defining the macro

stack $=\{$ push ; stack ; pop $\}$

We used this stack in a solution of the well-known puzzle called the 'towers of Hanoi'. In this puzzle a tower of blocks of different sizes has to be moved to another position, with the following constraints: First, blocks can be moved only one at the time. Second, it is not allowed to put a larger block on top of a smaller one. Third, only three positions for a tower can be used.

In figure 1 there are three legal moves: $\mathrm{B} 3$ to tower 2, B4 to tower 1 and B4 to tower 2 . Moving B3 to tower 3 is illegal, because $\mathrm{B} 3$ is larger than $\mathrm{B} 4$.

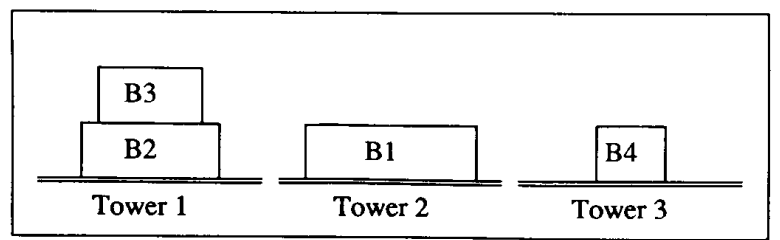

Figure 1. Hanoi towers.
The three towers are modelled as stacks with operations get and put. These actions have two parameters, the number of the stack and the current height of the tower:

tower $(T, h)=\{$ put $(T, h) ;$ tower $(T, h+1) ; \operatorname{get}(T, h)\}$

The operations place (T) puts a block on top of tower $T$. Because the height of the tower varies, place $(T)$ is defined as a choice between the different puts. Which of these is chosen is determined by the macros for tower T. An operation pick (T) is defined likewise.

$$
\begin{aligned}
& \text { place }(T)=<i=1 . . n: \operatorname{put}(T, i)> \\
& \text { pick }(T)=<i=1 . . n: \operatorname{get}(T, i)>
\end{aligned}
$$

Moving a single block from tower $\mathrm{T}$ to tower $\mathrm{Q}$ is now trivial:

$$
\text { smove }(T, Q)=\operatorname{pick}(T) ; \operatorname{place}(Q)
$$

We have now come to the point were the puzzle can be solved. The problem was: how to move the blocks from tower 1 to tower 2, according to the rules? The solution is recursive: first, we move all the blocks except the bottom one to tower 3 , then we move the bottom block to tower 2 , and the rest of the blocks from tower 3 to tower 2 .

The macro definition below describes how to move the upper $\mathrm{n}$ blocks of tower $\mathrm{T}$ to tower $\mathrm{Q}$. Tower $\mathrm{R}$ is the third tower.

$$
\begin{gathered}
\text { move }(\mathrm{n}, \mathrm{T}, \mathrm{Q}, \mathrm{R})= \\
\text { move }(\mathrm{n}-1, \mathrm{~T}, \mathrm{R}, \mathrm{Q}) ; \\
\text { smove }(\mathrm{T}, \mathrm{Q}) ; \\
\text { move }(\mathrm{n}-1, \mathrm{R}, \mathrm{Q}, \mathrm{T})
\end{gathered}
$$

$$
\text { move }(1, T, Q, R)=\text { smove }(T, Q)
$$

The path expressions Q1..Q7 are only macro definitions. In order to get the basic actions executed in the required order, we have to supply the rules governing the stack behaviour of the towers, using the macros defined before.

$$
\text { * } \mathrm{T}=1 . .3: \text { tower }(\mathrm{T}, 1) \text { ? }
$$

We can now give a path expression command to build an initial tower 1 and a command to move it to tower 2 .

$$
\text { place (1)! ; place (1)! ; place (1)! }
$$

move $(3,1,2,3)$ !

An implementation of this solution was made with a robot moving the blocks. In this implementation, the put and the get were defined as macros consisting of a series of elementary robot commands like 'moveto', 'grab' and 'drop'.

\section{Implementation aspects}

As mentioned before, a path expression is represented by a binary tree obtained by parsing the expression. Operators in the expression correspond to branching-nodes in the tree. The leafs are the end-nodes in the tree and represent the actions or macros. Infinite repetition is represented by letting a node branch back to itself. Several attributes are attached to nodes of the parse tree. Subexpressions that may lead to an empty evolution are marked as nullable. Other attributes are the activator/acceptor indication, the minimum and maximum number of repetitions and input/output redirection information. 
At runtime the dynamic state of the path expressions is maintained in a separate state tree. This state tree reflects the structure of the parse tree, but contains only the nodes which are enabled or in execution. Moreover, nodes of the state tree are removed when they become superfluous. A selection-node becomes useless after the choice has been made, and a sequence- and parallel-node become useless when the left respectively the first branch has been terminated. In this manner a minimal tree is maintained. The state tree permanently grows and shrinks when nodes are enabled, respectively disabled. Nodes corresponding to a repetition or macro substitution are only added as far as needed, so an infinite expansion is avoided. This kind of lazy expansion is both efficient and crucial in case of infinite or recursive specifications.

In order to decide which actions have to be started (at state changes) state information about the individual paths must be combined.

For each action-name the following state information is globally collected:

- a set passive-in consisting of the paths which contain the action as acceptor.

- a set disabled-in consisting of the paths in which all acceptors are disabled, or in other words in which no acceptor has been enabled. (This set is a subset of the set passive-in.)

a set active-in consisting of the paths in which an activator is enabled.

Actions must be activated by the controlling system when one of the following conditions holds:

1. The set disabled-in is empty and the set active-in is not empty (one or more activators are enabled and no paths inhibit the occurrence). Any path may be chosen from the set active-in to deliver the activator which will generate the occurrence.

2. The set disabled-in only contains a single path and the same path also appears in the set active-in (a path contains enabled activators as well as disabled acceptors, however, other paths do not inhibit the action).

If for an action the set disabled-in contains two or more paths surely no occurrence of this action is allowed.

When it is decided to start an action, all participating paths have to adapt their states. After the paths have reached their new state, the whole procedure is repeated until no action can be started any more. Thereafter only termination of actions and changes in predicates due to asynchronous events, or changes in the set of path expressions can trigger new activations. For more details we refer to [4].

\section{SySTEM BehaVIOUR MODELLING}

\section{A. Representing Finite State Machines}

Finite state machines are well known as a modelling method for a broad class of systems. We will show here how finite state machines can be represented in terms of path expressions.

A finite state machine is a directed graph. The nodes are called states and the edges are called transitions. One of the states is the start state. An execution of the machine is a walk through the graph, beginning in the start state and according to the edges.
For every transition from $S_{i}$ to $S_{j}$ we can define a condition $\mathrm{C}\left(\mathrm{S}_{\mathrm{i}}, \mathrm{S}_{\mathrm{j}}\right)$ that has to be true before the transition can be made, and an action $T\left(S_{i}, S_{j}\right)$ that is activated or accepted when the transition is made.

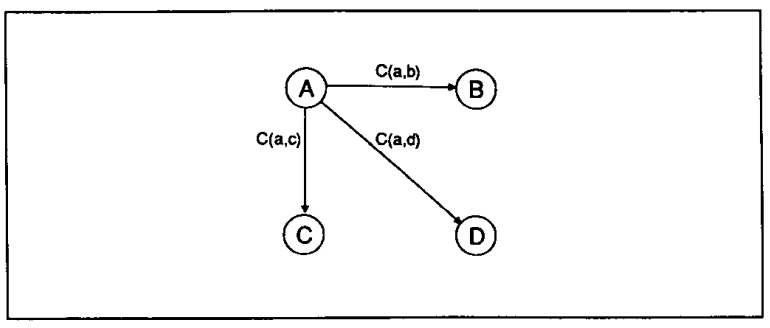

Figure 2. Transitions of one state

Consider figure 2. This part of a finite state machine can be represented with the following macro definition:

$$
\begin{aligned}
A= & \text { IF C (a,b) THEN T(a,b);B } \\
& \text { IF C(a,c) THEN T(a,c);C } \\
& \text { IF C (a,d) THEN T(a,d);D }
\end{aligned}
$$

Path expression R1 offers a conditional choice between three options. As soon as one of the conditions becomes true, the transition in question becomes enabled. Now what happens depends on the way the macro was used, as activator $\mathbf{A}$ ! or as acceptor A?. If the macro was used as A!, the transition action $T$ can be executed if the other rules allow it. If it was used as A?, the transition action becomes enabled.

If more than one of the conditions is true and the macro was used as $A$ !, the choice between the $T(a, x)$ is made nondeterministically, possibly influenced by the other rules. If the macro was used as A?, the choice is made by the environment (i.e. the other rules).

When the choice is made in favour of a transition to state $X$ the transition action $T(a, x)$ is executed, followed by macro $X$, which is defined completely analogous to $A$.

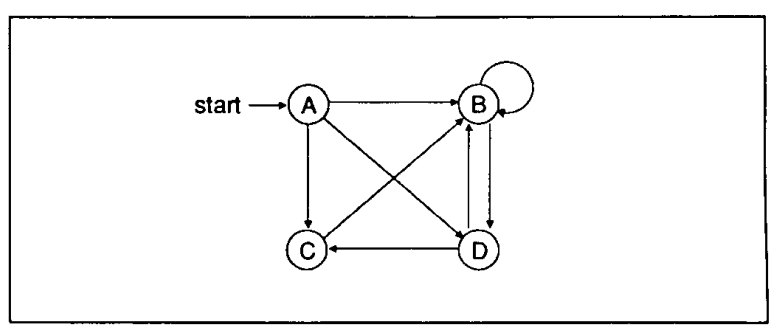

Figure 3. Simple finite state machine

The finite state machine of figure 3 is described by a set of four macro definitions, one for each state. State A has the same transitions as the example state $A$ from figure 2 , so we describe it with R1. Notice that the transition from $B$ back to $B$ gives no problems. 


$$
\begin{aligned}
& \mathrm{A}=\quad \text { IF C(a,b) THEN T(a,b);B } \\
& \text {, IF C }(a, c) \text { THEN T }(a, c) ; C \\
& \text {, IF C(a,d) THEN T(a,d);D } \\
& \mathrm{B}=\quad \text { IF } \mathrm{C}(\mathrm{b}, \mathrm{b}) \text { THEN T(b,b);B } \\
& \text {, IF C(b,b) THEN T(a,b);D } \\
& \mathrm{C}=\quad \text { IF C (c,b) THEN T(c,b);B } \\
& \begin{array}{l}
\mathrm{D}=\quad \text { IF C(d,b) THEN T(d,b);B } \\
\text { IF C(d,c) THEN T(d,c);C }
\end{array}
\end{aligned}
$$

To make an 'active' finite state machine, simply supply the rules R1..R4 and a command 'A!'. To make an 'accepting' finite state machine, simply supply the rules $R 1$..R4 and a rule 'A?'.

The representation of a finite state machine is given above in its most extensive form. Generally, not all transitions are subject to conditions, and not all transitions execute a transition action.

The representation can also be extended by actions that are executed when the machine is in a given state, in addition to the transition actions.

We can now, for state $\mathrm{A}$ of the machine above, get an expression like

$$
A=\operatorname{In}(A) ;(T(a, b) ; B, T(a, c) ; C, T(a, d) ; D)
$$

The conditions could also be given by separate rules. If, for example, all transitions to $\mathrm{A}$ depend on a condition $\mathrm{C}$, we can add the rule

\{ IF C THEN In(A) \}

This rule is independent from the way $\mathrm{A}$ is reached.

A finite state machine may thus interact with other rules, specifying additional parts of their behaviour.

At this point the power of path expressions may become clear. Different finite state machine models are easily combined by joining their path expression representation. Or, in other words, complex behaviour can be disentangled into a combination of rules and commands, which describe partial behaviour and are possibly based on simple finite state machines.

Whereas a single finite state machine only describes sequential behaviour, parallel behaviour of multiple finite state machines can be easily obtained by the parallel composition of their individual path expression representations.

The modelling approach as described here has been used in a bio-medical application [5]. This application is a high-level control system for the artificially stimulated walking of patients with paralysis of both legs. Different finite state models cover partial aspects like the walking sequence, use of crutches and foot-sole pressure detectors. They are combined in a path expression rulebase. Although the control is only simulated graphically, the results demonstrate that the method can in principle be applied in practice. Because the PBOS system makes it possible to experiment with the control rules, the system appears to be very useful for modelling purposes.

\section{B. Modelling Example}

Let us consider the operation of a group of elevators as another modelling example.
We will first describe the fact that elevators go up and down, and that they stop at certain floors. Then we will add rules that force the lifts to service requests that are made. Eventually we will specify rules to force the lifts to service requests in the correct order.

We assume that the building has floors $0 . \mathrm{N}$ and that there are M elevators.

The movements of elevator i can be described recursively by means of the following macro's:

$$
\begin{aligned}
\operatorname{lift}(\mathrm{i}, 0)= & \operatorname{stop}(\mathrm{i}, 0) ; \operatorname{up}(\mathrm{i}, 0) ; \operatorname{lift}(\mathrm{i}, 1) \\
\operatorname{lift}(\mathrm{i}, \mathrm{j})= & (\operatorname{stop}(\mathrm{i}, \mathrm{j}), \operatorname{pass}(\mathrm{i}, \mathrm{j})) ; \\
& (\operatorname{up}(\mathrm{i}, \mathrm{j}) ; \operatorname{lift}(\mathrm{i}, \mathrm{j}+1), \operatorname{dn}(\mathrm{i}, \mathrm{j}) ; \operatorname{lift}(\mathrm{i}, \mathrm{j}-1)) \\
\operatorname{lift}(\mathrm{i}, \mathrm{N})= & \operatorname{stop}(\mathrm{i}, \mathrm{N}) ; \operatorname{dn}(\mathrm{i}, \mathrm{N}) ; \operatorname{lift}(\mathrm{i}, \mathrm{N}-1)
\end{aligned}
$$

A group of $M$ elevators, initially at ground level (assume that floor 0 is the cellar), is specified by an activator rule:

$$
[i=1 \ldots M: \operatorname{lift}(i, 1)] \text { ! }
$$

In order to restrict their behaviour according to specific rules we will need the following macro definitions, which represent sets of alternative movements.

$$
\begin{aligned}
& \operatorname{up}(i)=\langle j=0 . . N-1: \operatorname{up}(i, j)> \\
& \operatorname{dn}(i)=\langle j=1 . . N: \operatorname{dn}(i, j)>
\end{aligned}
$$

The actual movements of the elevators will depend upon 'inside requests' made by pushing buttons in the lift cabin and 'outside requests' made by pushing buttons next to the lift doors. Assume the state of the former are given by predicates $\operatorname{Req}(\mathrm{i}, \mathrm{j})$, and of the latter by upReq(j) and $d n R e q(\mathrm{j})$. If one of these predicates holds, the according requests is made but not yet serviced.

In order to avoid detailed expressions we define also two compound predicate functions:

$$
\begin{aligned}
& \text { goUp }(i) \equiv \operatorname{Req}(i, j), \text { upReq }(j) \text { or } d n \operatorname{Req}(j) \text { holds for some } j \\
& \operatorname{goDn}(\mathrm{i}) \equiv \operatorname{Req}(\mathrm{i}, \mathrm{j}) \text {, upReq(j) or dnReq(j) holds for some } j \\
& \text { above the floor where elevator } i \text { currently is. }
\end{aligned}
$$

The first behavioural rule concerns the cases for which it makes sense to let an elevator move up or down:

$$
\begin{aligned}
* i= & 1 . \text { M : } \\
& \{\text { IF goUp(i) THEN up(i), } \\
& \text { IF goDn(i) THEN dn(i) }\}
\end{aligned}
$$

The second behavioural rule concerns a well-known servicing discipline related to elevators: requests in the current direction have to be served all before the elevator changes direction. When, for example, the elevator i moves upwards the elevator may not start going downwards as long as goUp(i) remains true. This kind of persistent behaviour can be expressed by the rules:

$$
\begin{aligned}
& * \mathrm{i}=1 . . \mathrm{M}:\{\text { IF NOT goDn(i) THEN }\{\text { up(i) }\} \text {; } \\
& \text { IF goDn(i) AND NOT goUp(i) THEN nil \} } \\
& *_{i}=1 . . M:\{\text { IF NOT goUp(i) THEN }\{\text { dn(i) }\} \text {; } \\
& \text { IF goUp(i) AND NOT goDn(i) THEN nil ] }
\end{aligned}
$$

Consider rule $\mathbf{S 8}$. The precondition for starting a repetition \{up(i)\} is that goDn(i) does not hold. Once started (in which 
case goUp(i) must hold), goDn(i) may become true without disabling the sequence of up-movements any more. Only when goUp(i) becomes false the sequence ends (forced by enabling an empty path which always 'occurs').

So far we have not yet formulated when an elevator can pass a floor, and when it has to stop. There are a few different cases when a stop on floor $\mathrm{j}$ has to be made:

1 when a request $\operatorname{Req}(\mathrm{i}, \mathrm{j})$ was made inside the elevator.

2 when a request $\operatorname{dnReq}(\mathrm{j})$ was made and the elevator is moving in downward direction.

3 when a request dnReq(j) was made and there are no requests to go further up. If there are requests to go further up, they are serviced first.

Of course, cases 2 and 3 have their symmetric counterpart for upReq(j).

To see if the elevator is going up or down we compare the moment of activation of the last up and down:

goingUp(i) $\equiv$ TACT_up(i) AFTER TACT_dn(i)

goingDn(i) $\equiv$ TACT_dn(i) AFTER TACT_up(i)

The criterion to stop at or pass by some floor can be formulated as:

$$
\begin{aligned}
& \text { * } \mathrm{i}=1 . . \mathrm{M}, \mathrm{j}=1 . . \mathrm{N}-1 \text { : } \\
& \text { IF } \operatorname{Req}(\mathrm{i}, \mathrm{j}) \mathrm{OR} \\
& \text { (dnReq(j) AND } \\
& \text { (goingDn(i) OR NOT goUp(i))) OR } \\
& \text { (upReq(j) AND } \\
& \text { (goingUp(i) OR NOT goDn(i))) } \\
& \text { THEN stop }(\mathrm{i}, \mathrm{j}) \text { ELSE pass }(\mathrm{i}, \mathrm{j})
\end{aligned}
$$

Although the rules given so far - if combined - force the elevators to operate in a correct manner, the restrictions do not yet lead to optimal behaviour in an economical sense. All elevators will compete in fulfilling outside requests. It is possible to specify rules to let the lifts co-operate but this falls outside the scope of this introductory exposition.

\section{ConClusions}

In this paper it is shown how realtime system behaviour can be formulated by means of multiple, interacting path expressions, each describing some part or aspect of the total system. The interference between path expression which is based on common action names, provides an elegant way of multi-way synchronization. In process-algebra-type languages, which have much in common with path expressions, synchronization is typically based on synchronous communication between processes (see [6]). This type of interaction provides only twoway synchronization.

Traditionally, path expressions are used to specify only the restrictions on the order of operations that are performed by parallel processes. Only in some cases path expressions are used to specify processes themselves. In COSY [3] there is a separation between path expressions specifying rules and path expressions specifying commands. Our way of using path expressions, where an expression can be a rule, a command or a mixture of both, gives a large additional flexibility, leading to a very natural description of system components, which closely reflects the way these components interact.

The development of the prototype system PBOS has demonstrated that path expression specification naturally integrates with a realtime, multitasking control system. The path expression based command language of PBOS offers a flexible, interactive way of specifying system behaviour.

Experience with the prototype system so far is based on several example problems. It appears that synchronization schemes derived from daily life such as traffic lights or elevators, of which the rules are well-known, are easily modelled by path expressions. It is a striking experience to see how rigourously the action control system follows the behaviour of a system, as formulated by the rules.

The incremental way in which path expression rules may be specified turns out to be of great value. Rules concerning mutual exclusion of actions or precedence relations are added (or removed) with ease. This makes the system very flexible.

The macro-facilities and the structuring of rules according to the hierarchy of command shell processes are essential with respect to managing complexity. Further ways of modularization may be obtained by extending on these structuring methods.

Presently we are applying our method in the area of robot control, by using PBOS as a steering mechanism handling the high-level, global control steps. As a demonstration we let a robot move blocks of the towers of Hanoi puzzle (see section 4B).

Another application that has been investigated concerns a biomedical control system. In this application artificially stimulated human walking is controlled according to multiple interacting finite state models (see section 5A).

Because the rulebase can be modified interactively and rules are applied automatically, PBOS makes it easy to experiment on-line with different path expression rules. Therefore the system turns out to be an useful modelling tool for system behaviour. Path expressions appear to be well suited to add intelligence into realtime, parallel systems, at a global level.

Similarities exist between path expression programming and logic programming languages like PROLOG. In both cases rules are given which specify the desired solution. A PROLOG program, however, results in the derivation of assertions, whereas the path expression control results in the derivation of allowed actions. Logic programming languages are generally used in the context of knowledge or expert systems. These systems are primarily intended for logic reasoning. However, more and more they are applied too for action planning of intelligent autonomous systems [7]. This kind of usage might be termed 'logistic reasoning' rather than logic reasoning. The proposed realtime system control based on path expressions can be viewed as a rule-based inference mechanism for action planning system. In that sense, our algebraic programming method could be characterized as logistic programming, as opposed to logic programming.

\section{REFERENCES}

[1] R.H.Campbell, A.N.Habermann, The specification of process synchronization by path expressions,

Lecture Notes in Computer Science, 16, Springer Verlag, 1974

[2] S. Andler

Predicate Path Expressions,

Conference Record of the 6th Annual ACM symposium on principles of programming languages, 1979. 
[3] P.E. Lauer, P.R. Torrigiani and M.W. Shields,

COSY - A system specification language based on paths and processes,

Acta Informatica 12, pp. 109-158, 1979.

[4] A.L. Schoute

Path expression based Operating Systems, Memorandum INF-

88-68, University of Twente, Dept. of Informatics, 1988.

[5] H. Jonkers,

High-level control of FES-assisted walking using path

expressions, Masters Degree Thesis, University of Twente,

Dept. of Informatics, 1990.

[6] J.J. Luursema

An overview of some methods for the high-level specification

of synchronization, Memorandum INF-88-50, University of

Twente, Dept. of Informatics, 1988.

[7] K. Fischer,

Knowledge-Based Task Planning for Autonomous Robot

Systems, Proceedings of the Conference on Intelligent

Autonomous Systems 2, Amsterdam, Elsevier Science

Publishers, 1989. 